(C) 2014 IEEE. Personal use of this material is permitted. Permission from IEEE must be obtained for all other uses, in any current or future media, including reprinting/republishing this material for advertising or promotional purposes, creating new collective works, for resale or redistribution to servers or lists, or reuse of any copyrighted component of this work in other works. 


\title{
Estimating Body-Fixed Frame Velocity and Attitude from Inertial Measurements for a Quadrotor Vehicle
}

\author{
Guillaume Allibert ${ }^{1}$ and Dinuka Abeywardena ${ }^{2}$ and Moses Bangura ${ }^{3}$ and Robert Mahony ${ }^{3}$
}

\begin{abstract}
A key requirement for effective control of quadrotor vehicles is estimation of both attitude and linear velocity. Recent work has demonstrated that it is possible to measure horizontal velocities of a quadrotor vehicle from strap-down accelerometers along with a system model. In this paper we extend this to full body-fixed-frame velocity measurement by exploiting recent work in aerodynamic modeling of rotor performance and measurements of mechanical power supplied to the rotor hub. We use these measurements in a combined attitude and velocity nonlinear observer design to jointly estimate attitude and bodyfixed-frame linear velocity. Almost global asymptotic stability of the resulting system is demonstrated using Lyapunov analysis of the resulting error system. The performance of the observer is verified by simulation results.
\end{abstract}

\section{INTRODUCTION}

Inertial measurement systems have a long history of being used for estimating the orientation of aerospace vehicles such as spacecrafts, aircrafts and missiles [5]. Most orientation estimators are based on the principle of observing known vectorial directions. For micro-aerial vehicles such as quadrotors, the two most commonly used vectorial measurements are the gravity and Earth's magnetic fields. In earlier work, Mahony et. al [10] showed that with just one vectorial measurement along with angular velocity can be used to estimate roll and pitch angles and gyroscope biases. This approach has been applied extensively in practice for attitude estimation in quadrotor vehicles using the accelerometers as a gravity estimate, even though the assumptions do not hold exactly in this case [12]. In particular, if the inertial accelerations of the vehicle are significant, then they need to be subtracted from the accelerometer measurement to obtain the true gravity vector measurement. Global Positioning Systems (GPS) can be employed for this purpose [6] but high rate absolute position and velocity measurements are a luxury that most mobile robots operating in urban and indoor environments lack. Recent investigations into the dynamics of quadrotor vehicles have revealed that the horizontal drag force affecting a quadrotor vehicle is proportional to the horizontal body frame velocity [3], [4], [11] and that this force can be effectively measured using a triad of body mounted accelerometers [12]. As a natural extension of this,

\footnotetext{
${ }^{1}$ Guillaume Allibert is with I3S-CNRS, Université de Nice Sophia Antipolis, France. Allibertai3s.unice.fr

${ }^{2}$ Dinuka Abeywardena is with Centre for Autonomous Systems, University of Technology, Sydney, Australia. Dinuka. Abeywardena@ieee.org

${ }^{3}$ Moses Bangura and Robert Mahony are with Research School of Engineering and Information Sciences, Australian National University, Canberra, Australia. Moses.Bangura@anu.edu.au, Robert. Mahonyeanu.edu.au
}

other authors have demonstrated that these accelerometer measurements can be combined with body mounted gyroscope measurements to obtain an estimate of the horizontal velocity of the quadrotor vehicle, along with its attitude [1], [9].

In this paper we consider the problem of designing a nonlinear observer for the full body-fixed-frame velocity along with the attitude of a quadrotor vehicle. The paper makes two principal contributions: Firstly, we show how aerodynamic modeling of the rotors can be used along with the accelerometer measurements and measurements of rotor speed and motor shaft torque available on some electronic speed controllers, to measure the full body-fixed-frame velocity of a quadrotor vehicle. This measurement depends directly on accelerometer measurements and is always subject to high level of noise. To address the noise issues, we propose a nonlinear observer in the spirit of Hua [6], but posed in the body-fixed-frame. The second principal contribution of the paper is to use a matrix decomposition of the general $\mathbb{R}^{3 \times 3}$ matrix that appears in this observer design in order to identify the orthogonal part of the observer directly. This provides a direct estimation of the attitude of the vehicle without requiring a second stage orientation observer as was necessary in prior literature. The performance of the proposed observer is verified by simulation.

The paper has four sections in addition to the present introduction. In Section II we introduce the model and some mathematical definitions. In Section III we use momentum theory to show how the full body-fixed-frame velocity can be estimated from only an inertial measurement unit (IMU) and a suitable electronic speed controller (ESC). Section IV presents the proposed observer and provides a stability proof for its performance while Section V presents some simulation to verify the performance of the proposed observer.

\section{BACKGROUND}

This section introduces the kinematic model underlying the observer problem considered and defines some Lie theory terminology that will be used in the sequel.

Consider a quadrotor vehicle as a rigid airframe with four independent rotors. A body-fixed frame, denoted $\{B\}$, is attached to the vehicle, and an inertial frame $\{A\}$ is fixed to the ground. Following the usual convention in aerospace the $z$-axis of the inertial frame is pointing downwards and the body-fixed frame corresponds to the inertial frame when the vehicle is in hover and oriented to the north. Let $m$ denote the mass of the vehicle. Let $V \in\{B\} \equiv \mathbb{R}^{3 \times 1}$ denote the linear velocity and $\Omega \in\{B\} \equiv \mathbb{R}^{3 \times 1}$ denote the angular velocity 
of the body frame $\{B\}$ with respect to the inertial frame $\{A\}$ expressed in $\{B\}$. Let $R \in \mathrm{SO}(3)$ denote the rotation matrix representing the orientation of the body-fixed frame $\{B\}$ with respect to the inertial frame $\{A\}$. The gravitational acceleration expressed in the inertial frame $\{A\}$ is given by $g \vec{e}_{3}$ where $\vec{e}_{3}=[0 ; 0 ; 1]$ is the unit vector in the $z$-axis.

Since the mass $m$ of the airframe of a quadrotor is a scalar constant, we can write a scaled version of the linear dynamics of the vehicle as

$$
\dot{V}=-\Omega \times V+g R^{\top} \vec{e}_{3}-\frac{1}{m} T \vec{e}_{3}-\frac{1}{m} H,
$$

where $T \in \mathbb{R}$ denotes the vertical thrust and $H \in \mathbb{R}^{3 \times 1}$ the horizontal drag force generated by the rotors. It should be noted that for slow moving quadrotors, $H^{\top} \vec{e}_{3}=0$. More details of the thrust and drag terms are provided in Section III where we discuss the aerodynamics of a quadrotor in detail. In this paper, we will not be concerned with the attitude dynamics and there is no need to model them.

The second order linear kinematics of a quadrotor are given by

$$
\dot{V}=-\Omega \times V+g R^{\top} \vec{e}_{3}+a,
$$

where $a \in\{B\}$ is the specific acceleration of $\{B\}$ with respect to $\{A\}$ expressed in $\{B\}$. That is $a$ is the sum of all the exogenous accelerations applied to the rigid-body. Coriolis and gravitational accelerations are associated with the internal dynamics of the rigid-body and are not modeled by the specific acceleration. Clearly, the second order kinematics and the scaled dynamics are closely related. However, we draw an important distinction associated with the nature of the exogenous signals. In particular, the second order kinematics incorporate the specific acceleration $a$, which in our case can be measured using an inertial measurement unit (IMU), while the dynamics incorporate the forces $T$ and $H$ that we will model using an aerodynamic analysis. Trivially, one has

$$
a=-\frac{1}{m} T \vec{e}_{3}-\frac{1}{m} H .
$$

Given that $a$ is measured, then this equation provides direct measurements of $T$ and $H$. In practice, this measurement should be treated with care due to high noise levels in $a$ as well as possible bias effects. However, the information provided by this correspondence lies at the core of a number of recent works for velocity and attitude estimation for quadrotors [12], [11], [9], [1].

The system model that we consider consists of the second order linear kinematics along with the first order attitude kinematics

$$
\begin{aligned}
& \dot{V}=-\Omega \times V+g R^{\top \vec{e}_{3}}+a, \\
& \dot{R}=R \Omega_{\times},
\end{aligned}
$$

where the linear operator $(.)_{\times}$maps any vector in $\mathbb{R}^{3 \times 1}$ to its corresponding skew-symmetric matrix in $\mathfrak{s o}(3)$ such that $x_{\times} y$ is equal to the cross product $x \times y$ for all $x, y \in \mathbb{R}^{3 \times 1}$.

Define $|$.$| to be the Euclidian norm in \mathbb{R}^{3 \times 1}$ and $\|$.$\| to$ be the Frobenius norm in $\mathbb{R}^{3 \times 3}$. That is for all $A \in \mathbb{R}^{3 \times 3}$, $\|A\|=\sqrt{\operatorname{tr}\left(A^{T} A\right)}$, where $\operatorname{tr}($.$) is the trace.$
The set $\mathrm{SO}(3)$ denotes the special orthogonal Lie-group and $\mathfrak{s o}(3)$ denotes its Lie-algebra, the set of skew-symmetric matrices. Define $\mathbf{U}(3)$ to be the set of all invertible upper triangular including entries along the diagonal matrices in $\mathbb{R}^{3 \times 3}$. It is easily verified that $\mathbf{U}(3)$ is a Lie-group with Lie algebra $\mathfrak{u}(3)$ given by the set of all upper triangular matrices: that is including upper triangular matrices that are not invertible. Any invertible matrix $A$ has a unique decomposition into an orthogonal and upper triangular part

$$
A=U Q, \quad Q \in \mathrm{SO}(3), \quad U \in \mathbf{U}(3) .
$$

The factorisation is locally unique and globally unique if the diagonal elements of $U$ are required to be positive.

Observe that the Lie-algebra's $\mathfrak{s o}(3)$ and $\mathfrak{u}(3)$ span $\mathbb{R}^{3 \times 3}$ in a natural manner under the usual vector space addition of matrices. Let $\mathbb{P}_{\text {as }}$ (anti-symmetric) and $\mathbb{P}_{\text {ut }}$ (upper-triangular) denote the associated projections onto $\mathfrak{s o}(3)$ and $\mathfrak{u}(3)$ respectively. For any matrix $A \in \mathbb{R}^{3 \times 3}$ given by

$$
A=\left(\begin{array}{lll}
a_{11} & a_{12} & a_{13} \\
a_{21} & a_{22} & a_{23} \\
a_{31} & a_{32} & a_{33}
\end{array}\right)
$$

then one has

$$
\mathbb{P}_{\text {as }}(A)=\left(\begin{array}{ccc}
0 & -a_{21} & -a_{31} \\
a_{21} & 0 & -a_{32} \\
a_{31} & a_{32} & 0
\end{array}\right) \in \mathfrak{s o}(3),
$$

and

$$
\mathbb{P}_{\mathrm{ut}}(A)=\left(\begin{array}{ccc}
a_{11} & a_{12}+a_{21} & a_{13}+a_{31} \\
0 & a_{22} & a_{23}+a_{32} \\
0 & 0 & a_{33}
\end{array}\right) \in \mathfrak{u}(3) .
$$

It is easily verified that $\mathbb{P}_{\mathrm{ut}}^{2}=\mathbb{P}_{\mathrm{ut}}$ and $\mathbb{P}_{\text {as }}^{2}=\mathbb{P}_{\mathrm{as}}$. Moreover,

$$
\mathbb{P}_{\text {ut }}(A)+\mathbb{P}_{\text {as }}(A)=A,
$$

for any matrix $A$. It is straightforward to verify that $\mathbb{P}_{\text {as }}\left(\mathbb{P}_{\text {ut }}(A)\right)=0$ and $\mathbb{P}_{\text {ut }}\left(\mathbb{P}_{\text {as }}(A)\right)=0$.

\section{Aerodynamic Power And Estimation of $V$}

This section presents a lumped model for the aerodynamics of a quadrotor. The approach is based on recent work in measuring aerodynamic power for quadrotor applications [3]. The model is based on using simplified momentum theory to relate thrust, horizontal drag forces and aerodynamic power and combining these relationships with (2).

\section{A. Horizontal drag force}

There are several recent works [1], [4], [9], [11], [12] that have discussed and exploited models for horizontal drag forces in estimating attitude and velocity for quadrotor and other aerial vehicles. The horizontal forces associated with induced drag, blade flapping, and translational drag [4] all manifest as bilinear functions of horizontal velocity and thrust magnitude

$$
H=-T K_{r} V \in\{B\}
$$


where $T \in \mathbb{R}$ is the thrust,

$$
K_{r}=\left(\begin{array}{ccc}
\bar{c} & 0 & 0 \\
0 & \bar{c} & 0 \\
0 & 0 & 0
\end{array}\right)
$$

and $\bar{c}>0$ is a lumped parameter that models the combined linear drag coefficients. In contrast, parasitic drag depends quadratically on vehicle velocity and since we consider quadrotors operating in near hover conditions, this effect is negligible.

\section{B. Vertical thrust force}

Modeling the thrust or vertical force of a rotor is complex because of the dependence of thrust on the induced velocity $v^{i}$ of air passing through the rotor; that is air velocity at the rotor plane that is induced by action of the rotor in generating thrust and separate from the relative wind experienced due to the motion of the vehicle through the air.

We will assume that the aerodynamic conditions across all four rotors are similar and there is an 'average' rotor speed $\varpi$ that can be used to model the aerodynamic performance of the quadrotor using momentum theory. We will also assume a single average induced velocity $v^{i}$ for all four rotors. We will use vector notation for the induced velocity $v^{i} \in\{B\}$, however following the lead of classical helicopter books [8], only the vertical component of the induced velocity is considered in the aerodynamic model. That is

$$
v^{i}=v_{z}^{i} \vec{e}_{3}
$$

where $v_{z}^{i} \in \mathbb{R}$ is a scalar. We will assume that all rotors have the same flapping angles and generate the same aerodynamic drag components. These assumptions are reasonable if the quadrotor is flying in near hover conditions.

Consider a control volume for a single rotor shown in Figure 1. Momentum theory for a single rotor yields [8]

$$
T_{k}=2 \rho A v_{z}^{i} U
$$

where $T_{k}$ denotes the thrust associated with rotor $k, \rho$ is the density of air, $A$ is the area of a rotor disc, and

$$
U=\left|v^{i}-V\right|
$$

is the magnitude of the total wind velocity, including both vehicle velocity and induced velocity of the air through the rotor. Note that the wind due to the vehicle motion is $-V$, the negative of the vehicle motion. Let $c_{1}=8 \rho A_{\text {total }} \in \mathbb{R}$, then the total thrust generated by all four rotors is modeled by

$$
T=c_{1} v_{z}^{i} U
$$

Unlike the classical helicopter analysis found in books such as Leishman [8], where only the steady-state performance in the separate lift or drag directions is required, we will provide a combined model that includes transient effect for power in the rotor and both thrust and drag in a single model. The aerodynamic power $P_{a}$ supplied to the air consists of two components: vertical $P_{T}$ and horizontal $P_{H}$ power,

$$
P_{a}=P_{T}+P_{H}
$$

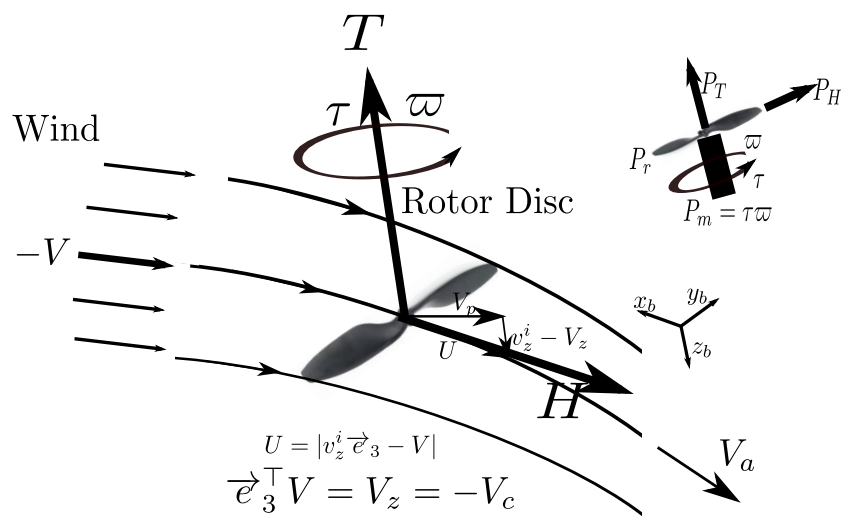

Fig. 1. Momentum Theory control volume and rotor hub showing the various powers for one of the rotors. In the control volume, we have the axis definition for $\{B\}$ which may not always align with the rotor tip path plane. $V_{c}=-V_{z}$ is the climb velocity described in the helicopter literature.

associated with providing thrust and overcoming drag respectively.

The power associated with motion of the air in the vertical direction is [8]

$$
P_{T}=T\left(v_{z}^{i}-V_{z}\right)
$$

where $T v_{z}^{i}$ is the induced power (required to operate the rotor and overcome induce aerodynamic forces) and $-T V_{z}$ is the power to climb. Note that due to the choice of axis, when $V_{z}<0$ the vehicle is ascending and $-T V_{z}$ is positive as expected. In the horizontal plane one has

$$
P_{H}=-H^{\top} V>0,
$$

where $H \in\{B\}$ is a vector quantity that lies uniquely in the horizontal plane and hence only the horizontal velocity contributes to the power $P_{H}$.

To account for losses such as tip loss and rotational wake, there is an efficiency factor between the mechanical power supplied and the aerodynamic power at steady state. We refer to this efficiency as the figure of merit (FoM) which is a number between 0 and 1 and is obtained from static thrust tests [4]. It follows that the mechanical power $P_{m}$, supplied to the rotor hub factors into

$$
P_{m}=P_{r}+\frac{1}{\text { FoM }} P_{a}
$$

where the power supplied to accelerate or decelerate the rotor is $P_{r}=I \varpi \dot{\varpi}$. If the torque on the rotor shaft is $\tau$, then the mechanical power supplied to the rotor hub is given by $P_{m}=\tau \varpi$. The torque $\tau$ can be measured by identifying motor parameters and measuring current. The rotor speed $\varpi$ is measured in the operation of all Electronic Speed Controllers (ESCs) and a complementary filter for estimating $\dot{\varpi}$ is provided in [3]. It follows that $P_{r}$ and $P_{m}$ can be measured, and once FoM is identified then $P_{a}$ can be treated as a measured variable.

Counting equations, one has two constraints from (4) (note that although this is a compound equation with three components, only the two associated with the horizontal rotor plane contain meaningful information), and one constraint 
from both (6) and (8) in six unknowns $V_{x}, V_{y}, V_{z}, v_{z}^{i}, H$, and $T$. However, adding the constraint measurement (2) that has three additional constraints ensures that this system of equations can be resolved algebraically.

Given that $a$ is measured, then $T, V_{x}$ and $V_{y}$ can be solved for directly using (2) and (4). This is the same approach used in [1], [4], [9], [11], [12] to measure horizontal velocity of the vehicle. From (4) and (9) and using $T, V_{x}$ and $V_{y}$ then $P_{H}$ is computed. It follows that using (7) and (10) then $P_{T}$ can be computed. With $P_{T}$ known, then (8) is used to compute $\left(v_{z}^{i}-V_{z}\right)$. Finally, this is used to compute the total wind velocity $U$ and substituted into (6) to compute $v^{i}=v_{z}^{i} \vec{e}_{3}$. Once $v_{z}^{i}$ is computed the $z$-axis velocity $V_{z}$ is available by substituting back into the known value of $\left(v_{z}^{i}-V_{z}\right)$.

The key outcome of this algebraic process is the computation of a measured value of the body-fixed frame velocity $V=\left(V_{x}, V_{y}, V_{z}\right)$. We emphasise that this "measurement" is independent of orientation of the vehicle, unlike the approximations made in prior work [1], [4], [9], [11], [12]. The assumptions on the aerodynamic conditions still limit applications to those where the vehicle is in hover or near hover conditions. However, the additional identification of $z$-axis velocity provides the potential for a more advanced observer design than developed in prior works.

\section{OBSERVER DESIGN}

In this section, a nonlinear observer for estimating the body-fixed-frame velocity for a quadrotor is proposed. The approach is based on deterministic Lyapunov design techniques and draws heavily from the approach pioneered in [6].

For a quadrotor vehicle with an inertial measurement unit (IMU), the angular velocity $\Omega$ and specific acceleration $a$ are available. If the same vehicle is equipped with electronic speed controllers (ESCs) that measure both rotor speed and combined torque to all four motors then the mechanical power supplied to the rotor hubs can be measured. As we have seen in Section III, this is sufficient to provide a measurement of the body-fixed-frame velocity $V \in\{B\}$ of the vehicle. If the IMU is also equipped with magnetometers then these can be used to provide an additional measurement

$$
\mu=R^{\top} \stackrel{\circ}{\mu} \in\{B\}
$$

for $\stackrel{\mu}{\mu} \in\{A\}$ the inertial "known" magnetic field. In practice, the magnetic field measurement is often corrupted by onboard magnetic fields and cannot be used for attitude estimation. For this reason we will initially develop the proposed observer in the case where the magnetic field is not available and only provide a remark about the case where $\mu$ is measured.

The goal of the observer design is to provide estimates $\hat{V} \in \mathbb{R}^{3}$ and $\hat{R} \in \mathrm{SO}(3)$ of the body-fixed-frame velocity and attitude of the quadrotor. We will distinguish between the true velocity $\stackrel{\circ}{V}(t)$ and the measured velocity $V(t)$ in the following theorem for the sake of clarity; although the deterministic stability analysis is based on the relationship $V(t)=\stackrel{\circ}{V}(t)$.
Assumption 1 Assume that the trajectory of the quadrotor is sufficiently smooth such that $\Omega(t), \dot{\Omega}(t), \dot{V}(t)$ and $\ddot{V}(t)$ are bounded signals.

Theorem 1 Consider system (3) with $\Omega$, a and $V$ measured. Consider the observer

$$
\begin{gathered}
\dot{\hat{V}}=-\Omega_{\times} \hat{V}+g X^{\top \vec{e}_{3}}+a-k_{1} \Delta_{1}, \\
\hat{V}(0)=V(0) \\
\dot{X}=\dot{U} \hat{R}+U \dot{\hat{R}}, \quad X(0)=U(0) \hat{R}(0),
\end{gathered}
$$

where $k_{1}>0$ is a scalar gain and $X=U \hat{R}$ is the uppertriangular orthogonal decomposition of a general matrix $X \in \mathbb{R}^{3 \times 3}$. The dynamics of $U$ and $\hat{R}$ are given by

$$
\begin{gathered}
\dot{U}=-k_{2} g U \mathbb{P}_{u t}\left(U^{-1} \vec{e}_{3}(\hat{V}-V)^{\top} \hat{R}^{\top}\right), \\
U(0)=I_{3} \\
\dot{\hat{R}}=\hat{R} \Omega_{\times}+k_{2} \hat{R} \Delta_{2}, \quad \hat{R}(0)=I_{3} \\
\Delta_{1}=\hat{V}-V \\
\Delta_{2}=-g \hat{R}^{\top} \mathbb{P}_{a s}\left(U^{-1} \vec{e}_{3}(\hat{V}-V)^{\top} \hat{R}^{\top}\right) \hat{R},
\end{gathered}
$$

where $k_{2}>0$ is a scalar gain. Suppose that Assumption 1 is satisfied, then for almost all initial conditions, the estimate $\hat{V}(t) \rightarrow \stackrel{\circ}{V}(t)$ and

$$
\hat{R}^{\top} \vec{e}_{3} \rightarrow R^{\top} \vec{e}_{3}
$$

Proof: Define a velocity error

$$
\tilde{V}=\hat{V}-V \text {. }
$$

The time derivative of $\tilde{V}$ is given by

$$
\begin{aligned}
\dot{\tilde{V}} & =\dot{\hat{V}}-\dot{V} \\
& =-\Omega_{\times} \tilde{V}+(X-R)^{\top} g \vec{e}_{3}-k_{1} \Delta_{1} .
\end{aligned}
$$

Define a candidate Lyapunov function

$$
\mathcal{L}:=\frac{1}{2} \tilde{V}^{\top} \tilde{V}+\frac{1}{2 k_{2}}\|X-R\|^{2} .
$$

The time derivative of $\mathcal{L}$ satisfies

$$
\begin{aligned}
& \dot{\mathcal{L}}=\tilde{V}^{\top}(X-R)^{\top} g \vec{e}_{3}-k_{1} \tilde{V}^{\top} \Delta_{1}+ \\
& \frac{1}{k_{2}} \operatorname{tr}\left((X-R)^{\top}\left(\dot{U} \hat{R}+k_{2} X \Delta_{2}\right)\right) \\
&=\underbrace{\operatorname{tr}\left(\tilde{V}^{\top}(X-R)^{\top} g \vec{e}_{3}\right)}_{\mathrm{A}}+ \\
& \underbrace{\frac{1}{k_{2}} \operatorname{tr}\left((X-R)^{\top}\left(\dot{U} \hat{R}+k_{2} X \Delta_{2}\right)\right)}_{\mathrm{B}}-\underbrace{k_{1} \tilde{V}^{\top} \Delta_{1}}_{\mathrm{C}}
\end{aligned}
$$

Since the rotation matrix $R$ is unknown, the innovation terms $\Delta_{2}$ and $\dot{U}$ must be chosen properly in order to cancel the expressions $\mathrm{A}$ and $\mathrm{B}$ in the previous equation while $\Delta_{1}$ is 
chosen to make $\dot{\mathcal{L}}$ negative. Considering just terms $\mathrm{A}$ and $\mathrm{B}$ from above, one has

$$
\begin{aligned}
\operatorname{tr} & \left(\tilde{V}^{\top}(X-R)^{\top} g \vec{e}_{3}\right)+\frac{1}{k_{2}} \operatorname{tr}\left((X-R)^{\top}\left(\dot{U} \hat{R}+k_{2} X \Delta_{2}\right)\right) \\
& =\operatorname{tr}\left((X-R)^{\top}\left(g \vec{e}_{3} \tilde{V}^{\top}+\frac{1}{k_{2}} \dot{U} \hat{R}+X \Delta_{2}\right)\right) \\
& =\frac{1}{k_{2}} \operatorname{tr}\left((X-R)^{\top}\left(k_{2} g \vec{e}_{3} \tilde{V}^{\top} \hat{R}^{\top}+\dot{U}+k_{2} U \hat{R} \Delta_{2} \hat{R}^{\top}\right) \hat{R}\right) \\
& =\frac{1}{k_{2}} \operatorname{tr}\left((X-R)^{\top} U \Gamma \hat{R}\right),
\end{aligned}
$$

where the matrix $\Gamma \in \mathbb{R}^{3 \times 3}$ is given by

$$
\Gamma=k_{2} g U^{-1} \vec{e}_{3} \tilde{V}^{\top} \hat{R}^{\top}+U^{-1} \dot{U}+k_{2} \hat{R} \Delta_{2} \hat{R}^{\top} .
$$

Since $X$ can be an arbitrary matrix, then the only choice to cancel terms A and B from (15) for all possible $X$ is to set $\Gamma \equiv 0$. Using the projectors defined in Section II, one can split $\Gamma$ into its components

$$
\Gamma=\mathbb{P}_{\mathrm{as}}(\Gamma)+\mathbb{P}_{\mathrm{ut}}(\Gamma),
$$

with

$$
\begin{aligned}
& \mathbb{P}_{\mathrm{as}}(\Gamma)=k_{2} g \mathbb{P}_{\mathrm{as}}\left(U^{-1} \vec{e}_{3} \tilde{V}^{\top} \hat{R}^{\top}\right)+k_{2} \hat{R} \Delta_{2} \hat{R}^{\top} \\
& \mathbb{P}_{\mathrm{ut}}(\Gamma)=k_{2} g \mathbb{P}_{\mathrm{lt}}\left(U^{-1} \vec{e}_{3} \tilde{V}^{\top} \hat{R}^{\top}\right)+U^{-1} \dot{U}
\end{aligned}
$$

since $\Delta_{2}=-\Delta_{2}^{\top}, \mathbb{P}_{\text {as }}\left(U^{-1} \dot{U}\right)=0$ and $\mathbb{P}_{\text {ut }}\left(k_{2} \hat{R} \Delta_{2} \hat{R}^{\top}\right)=$ 0 . Substituting (12d) for $\Delta_{2}$ and (12a) for $\dot{U}$, it is easily verified that $\mathbb{P}_{\mathrm{as}}(\Gamma)=0=\mathbb{P}_{\mathrm{lt}}(\Gamma)$ and hence $\Gamma \equiv 0$. Substituting (12c), one obtains

$$
\dot{\mathcal{L}}=-k_{1} \tilde{V}^{\top} \tilde{V} \leq 0
$$

Since the time derivative of $\dot{\mathcal{L}}$ is semi-negative definite and $\mathcal{L}$ is positive definite then $\tilde{V}$ and $X$ are bounded. In view of (13) and Assumption 1, one deduces that $\dot{\tilde{V}}$ is bounded and it follows that $\ddot{\mathcal{L}}$ is also bounded. This is sufficient to ensure that $\dot{\mathcal{L}}$ is uniformly continuous along trajectories of the system. Applying Barbalat's lemma [7] ensures the convergence of $\dot{\mathcal{L}} \rightarrow 0$ that implies the convergence of $\tilde{V}$ to 0 .

The same procedure is performed to prove that $\ddot{\tilde{V}}$ is bounded (since $\dot{\Omega}, \dot{\tilde{V}}, \dot{X}$ and $\dot{\hat{R}}$ are bounded) and consequently to demonstrate the uniform continuity of $\dot{\tilde{V}}$. Barbalat's lemma ensures the convergence of $\dot{\tilde{V}}$ to 0 which implies from (13) the convergence of $X^{\top} g \vec{e}_{3}$ to $R^{\top} g \vec{e}_{3}$.

Finally, substituting $X=U \hat{R}$, one sees that $\hat{R}^{\top} U^{\top} \vec{e}_{3} \rightarrow$ $R^{\top} \vec{e}_{3}$. Taking norms, one has $\left|U^{\top} \vec{e}_{3}\right| \rightarrow 1$, that is the $(3,3)$ entry of $U$ converges to unity and $U^{\top} \vec{e}_{3} \rightarrow \vec{e}_{3}$. It follows that $\hat{R}^{\top} \vec{e}_{3} \rightarrow R^{\top} \vec{e}_{3}$ by continuity.

Since $\hat{R}^{\top} \vec{e}_{3} \rightarrow R^{\top} \vec{e}_{3}$, then this observer will effectively identify the pitch and roll components of the attitude of a quadrotor. The remaining degree of freedom in the orientation, corresponding to yaw rotation around the $\vec{e}_{3}$ axis is unobservable without additional measurements. If magnetometer measurements are available, then the filter can be augmented with these measurements to obtain full estimation of the attitude by replacing (12b) by

$$
\dot{\hat{R}}=\hat{R} \Omega_{\times}+k_{2} \hat{R} \Delta_{2}-k_{3} \hat{R}\left(\mu \times \hat{R}^{\top} \stackrel{\circ}{\mu}\right)_{\times}, \quad \hat{R}(0)=I_{3} .
$$

Although the proof is beyond the scope of the present paper, it is relatively straightforward to show that as long as $\stackrel{\mu}{\mu}$ is not collinear with $\vec{e}_{3}$ then $\hat{R}(t) \rightarrow R(t)$ locally.

\section{SIMULATION RESULTS}

In this section, we illustrate through simulation results the performance and robustness of the proposed observer. Simulations are performed for a model of a quadrotor aerial vehicle. The complete observer scheme is shown in Figure 2.

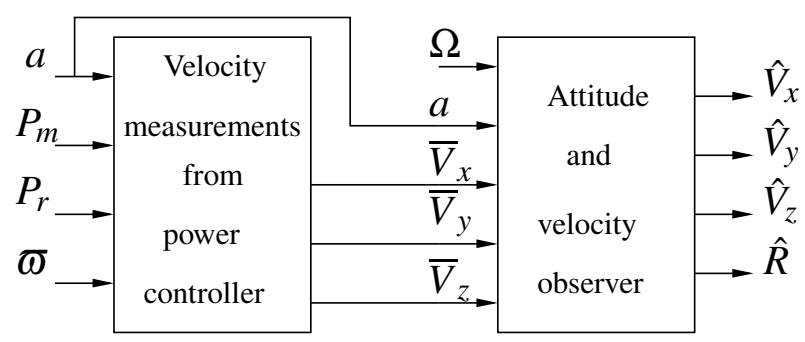

Fig. 2. Observer scheme

The trajectories of both $V(t), a(t)$ and $R(t)$ are generated assuming that the vehicle performs circular motion in the inertial frame combined with a sinusoidal changes of altitude. The vehicle's linear velocity given in the inertial frame is $v(t)=\left[0.5 \sin \left(\alpha_{1} t\right) ; 0.5 \sin \left(\alpha_{1} t+\frac{\pi}{2}\right) ; \sin \left(\alpha_{2} t\right)\right] \in\{A\}$ with $\alpha_{1}=\frac{1}{2}$ and $\alpha_{2}=\frac{1}{3}$. For the observer given by (11), the gains are $k_{1}=10$ and $k_{2}=1$. If $R_{x}(\theta), R_{y}(\theta), R_{z}(\theta)$ are the rotations about the $x, y, z$-axis rspectively by $\theta$, then the initial conditions are $\hat{R}(0)=R_{x}(10) R_{y}(-20) R_{z}(30)$ and $U(0)=[.500 ; .2 .30 ; .4 .5 .2]$, ensuring that $\hat{R} \in \mathrm{SO}(3)$ and $U$ is an upper triangular matrix. In the following, we assume that we can measure $P_{a}=F o M\left(P_{m}-P_{r}\right)$. In terms of practical implementation, [3] showed that $P_{a}$ can be measured from the current and $\varpi$ along with a complementary filter which estimates $\dot{\varpi}$ onboard an electronic speed controller board [2]. We also consider that the onboard IMU gives the specific acceleration and angular velocity measurements. In order to have a more realistic simulation, a random signal with a constant power spectral density is added to all inputs of the observer. The range of this signal is in accordance with the sensors used. For the accelerometer measurements, a high frequency noise of $200 \%$ the magnitude of typical accelerations experienced by such vehicles is added.

The noise in $\Omega$ of relative magnitude $20 \%$ is also added. For the $P_{a}$ used, the experimental results obtained in [3] show that the estimated values have relative noise that are under $10 \%$. Hence $10 \%$ noise was added to $P_{a}$. The performance of the proposed observer is shown in Figures 3 and 4 . The plots show the convergence and the performance of the proposed observer for the three body velocities. Note 
that despite the magnitude of noise in the input as well as in $\bar{V}$, the outputs of the observer are smooth and track the true real body velocities. Finally, Figure 4 shows the convergence of $\hat{R}^{\top} e_{3}$ to $R^{\top} e_{3}$ and the convergence of $U(3,3)$ to 1 .
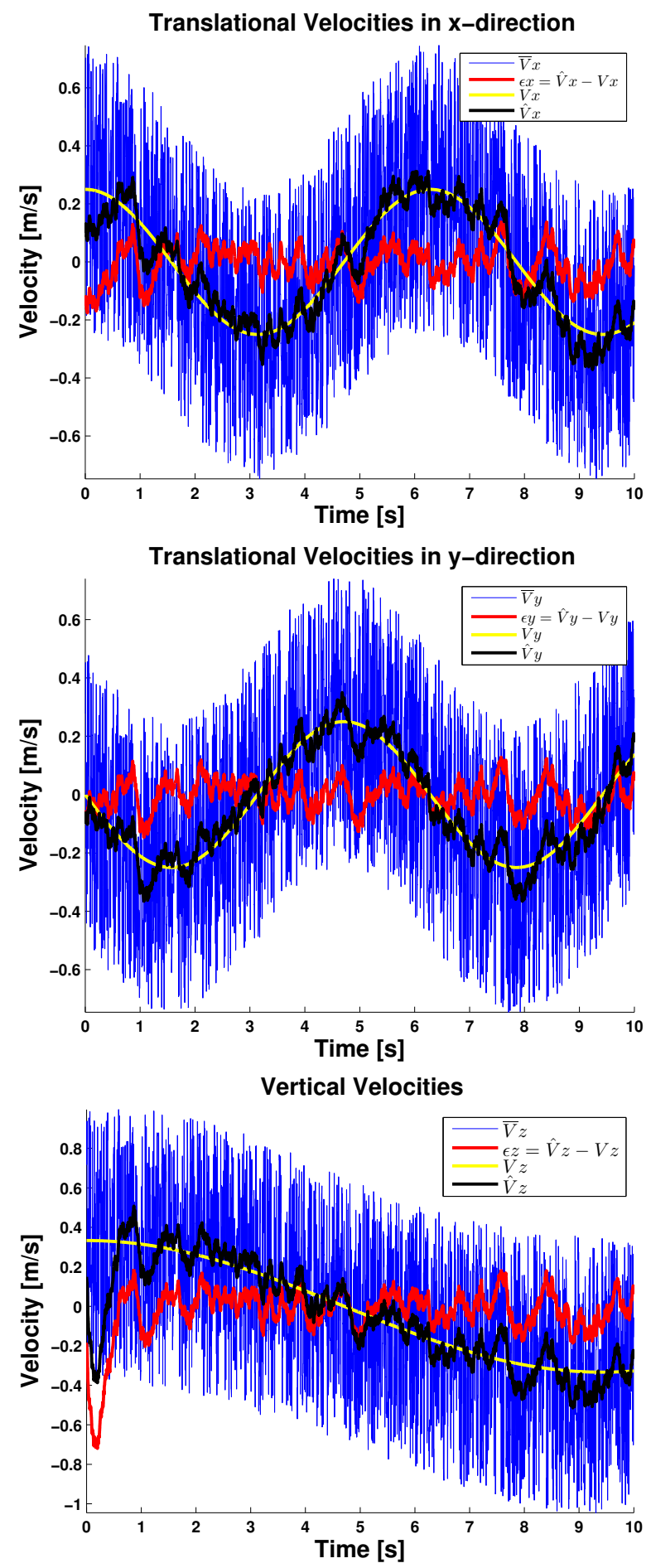

Fig. 3. Results of the simulated $V \in\{B\}$. With $V$ being the true velocity, we can see that despite the $200 \%$ noise in accelerometer measurements that is reflected in $\bar{V}$ determined algebraically as in Section III, the estimated velocities $\hat{V}$ track quite well the true velocity $V$. This is indicated by the small difference $\epsilon$ between estimated $\hat{V}$ and true $V$.
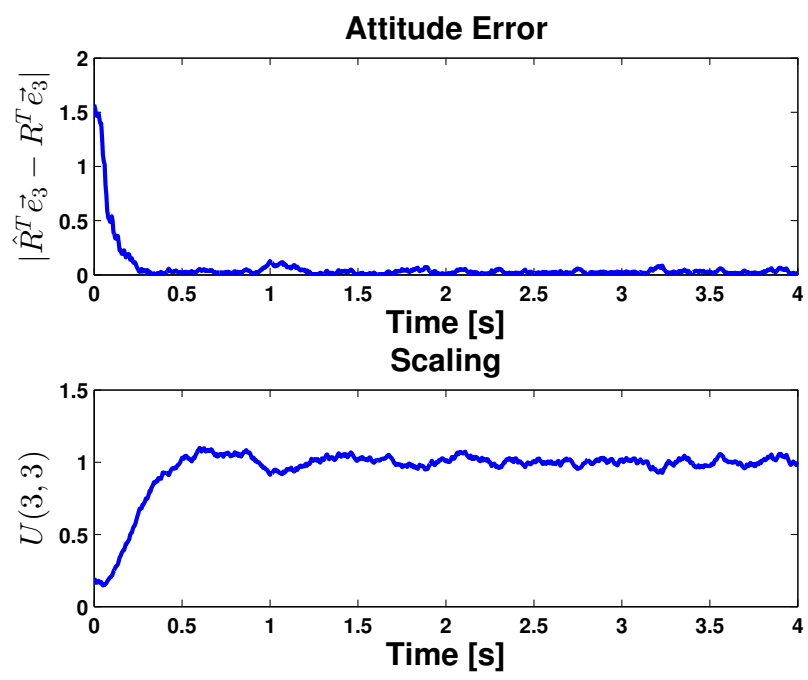

Fig. 4. Attitude error and scaling factor. We can see that the norm of the attitude error between estimated $\left(\hat{R}^{\top} \vec{e}_{3}\right)$ and true $\left(R^{\top} \vec{e}_{3}\right)$ converges to zero in less than 0.5 s which implies $\hat{R}^{\top} \vec{e}_{3} \rightarrow R^{\top} \vec{e}_{3}$. This performance is further reaffirmed by $U \vec{e}_{3} \rightarrow 1$.

\section{ACKNOWLEDGMENT}

This research was supported by the Australian Research Council through Discovery Grant DP120100316 "Integrated High-Performance Control of Aerial Robots in Dynamic Environments".

\section{REFERENCES}

[1] D. Abeywardena, S. Kodagoda, G. Dissanayake, and R. Munasinghe. Improved state estimation in quadrotors mavs. Robotics Automation Magazine, IEEE, 20(4):32 - 39, 2013.

[2] Autoquad Team. The AutoQuad ESC32 - a yet unseen electronic speed controller. http://autoquad.org/esc32/, 2012. [Online; accessed 7-Aug-2013].

[3] M. Bangura, H. Lim, H.J. Kim, and R. Mahony. Aerodynamic power control for multirotor aerial vehicles. In in Proc. IEEE Int. Conf. Robotics Automation, 2014.

[4] M. Bangura and R. Mahony. Nonlinear dynamic modeling for high performance control of a quadrotor. In in Proc. Australasian Conf. Robotics Automation, pages 3-5, 2012.

[5] J L Crassidis, F L Markley, and Y Cheng. Survey of nonlinear attitude estimation methods. Journal of Guidance, Control, and Dynamics, 30(1):12-28, 2007.

[6] M.D. Hua. Attitude estimation for accelerated vehicles using gps/ins measurements. Control Engineering Practice (Special Issue on Aerial Robotics), 18(7):723 - 732, 2010.

[7] H. K. Khalil. Nonlinear systems. Macmillan Publishing Company, 1992.

[8] J. G. Leishman. Principles of helicopter aerodynamics. Cambridge University Press, 2006.

[9] R.C. Leishman, J.C. Macdonald JR., R.W. Beard, and T.W. Mclain. Quadrotors and accelerometers: state estimation with an improved dynamic model. Control Systems Magazine, IEEE, 34(1):28 - 41, 2014.

[10] R. Mahony, T. Hamel, and J.-M. Pflimlin. Nonlinear complementary filters on the special orthogonal group. IEEE Trans. Autom. Contr., 53(5):1203-1218, 2008.

[11] R. Mahony, V. Kumar, and P. Corke. Multirotor aerial vehicles: Modeling, estimation, and control of quadrotor. Robotics Automation Magazine, IEEE, 19(3):20-32, 2012.

[12] P. Martin and E. Salaun. The true role of accelerometer feedback in quadrotor control. In in Proc. IEEE Int. Conf. Robotics Automation, pages $1623-1629,2010$. 